Supplement of Hydrol. Earth Syst. Sci., 24, 2731-2754, 2020

https://doi.org/10.5194/hess-24-2731-2020-supplement

(c) Author(s) 2020. This work is distributed under

the Creative Commons Attribution 4.0 License.

(c) (1)

Supplement of

\title{
Diagnosis of future changes in hydrology for a Canadian Rockies headwater basin
}

Xing Fang and John W. Pomeroy

Correspondence to: Xing Fang (xing.fang@usask.ca)

The copyright of individual parts of the supplement might differ from the CC BY 4.0 License. 
(a)

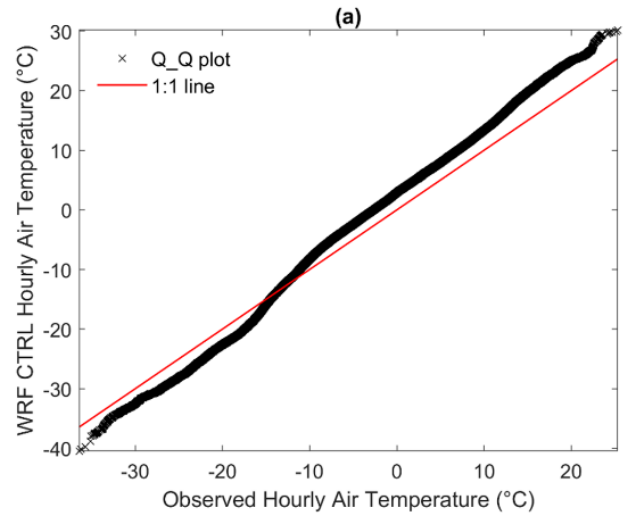

(c)

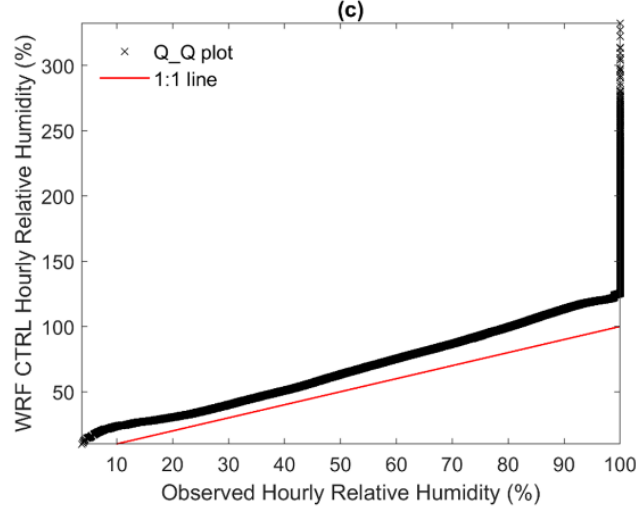

(e)

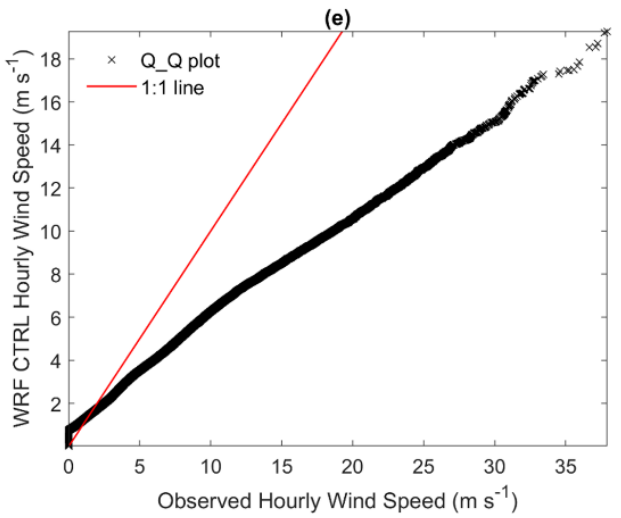

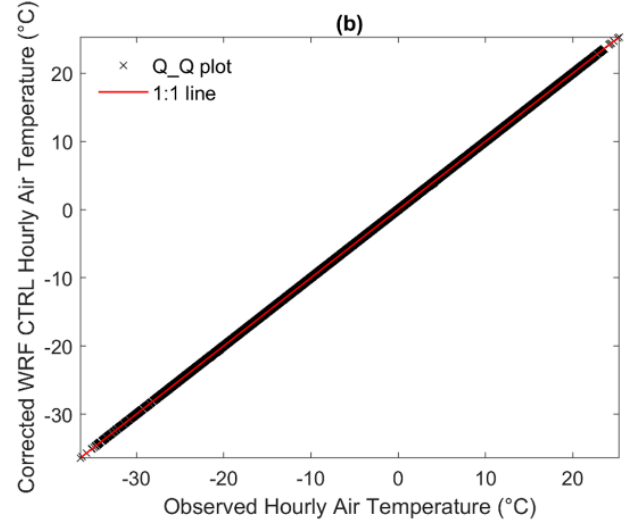

(d)

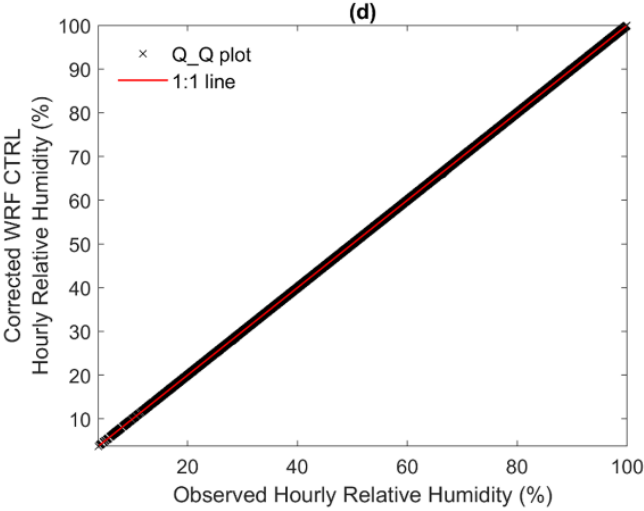

(f)

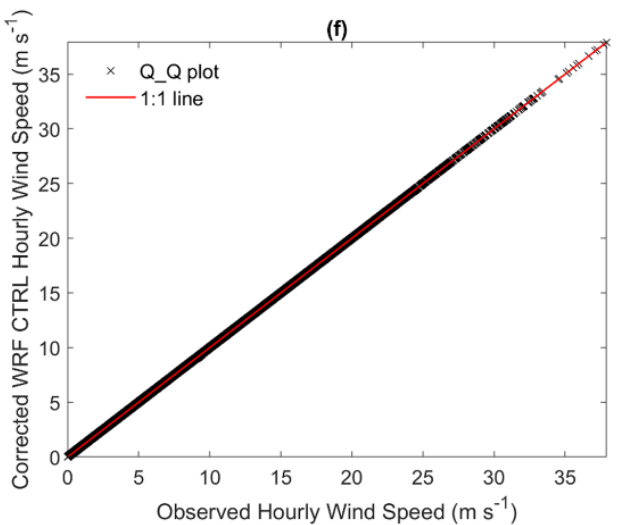

Figure S1. Quantile-quantile plots of observations and WRF CTRL outputs for Centennial Ridge station in MCRB: (a) WRF CTRL and observed air temperature, (b) corrected WRF CTRL and observed air temperature, (c) WRF CTRL and observed relative humidity (d) corrected WRF CTRL and observed relative humidity, (e) WRF CTRL and observed wind speed (f)

5 corrected WRF CTRL and observed wind speed, (g) WRF CTRL and observed incoming solar radiation (h) corrected WRF CTRL and observed incoming solar radiation, (i) WRF CTRL and observed precipitation (j) corrected WRF CTRL and observed precipitation. 
(g)

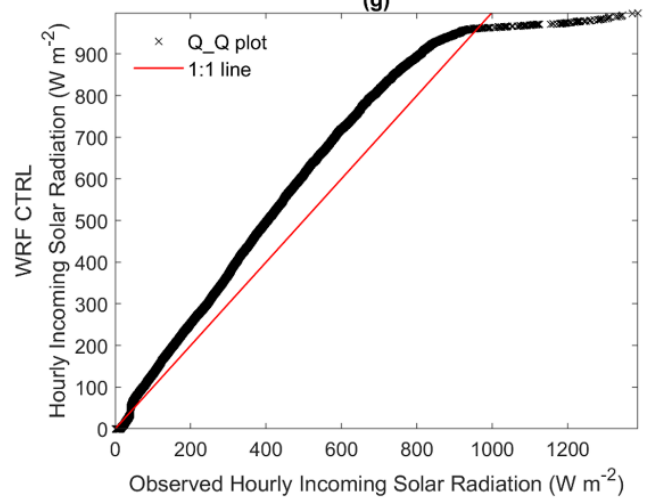

(h)

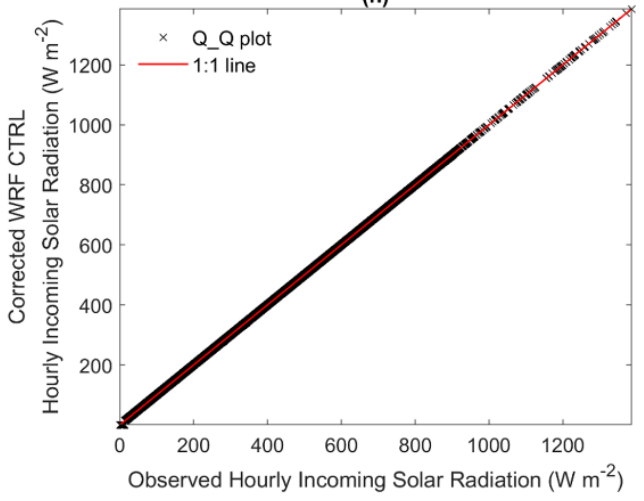

Figure S1. Continued. 


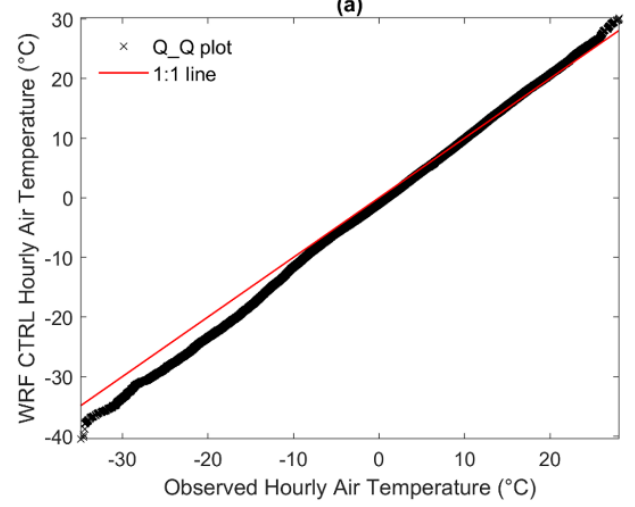

(c)

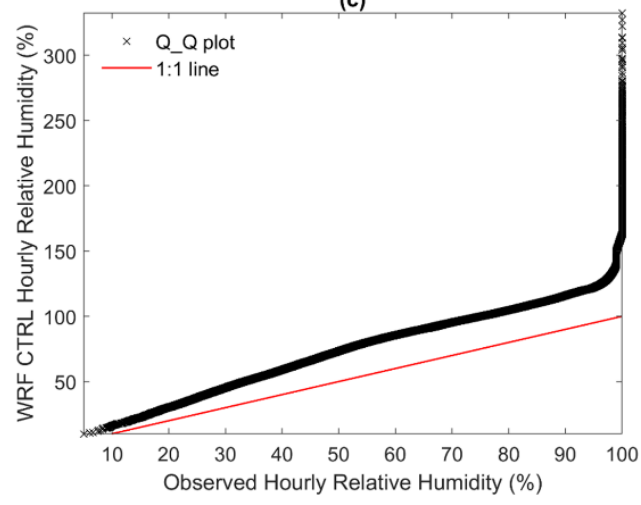

(e)

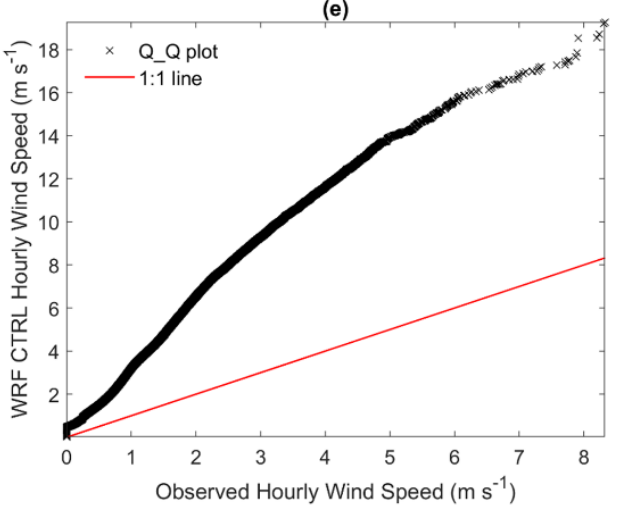

(b)

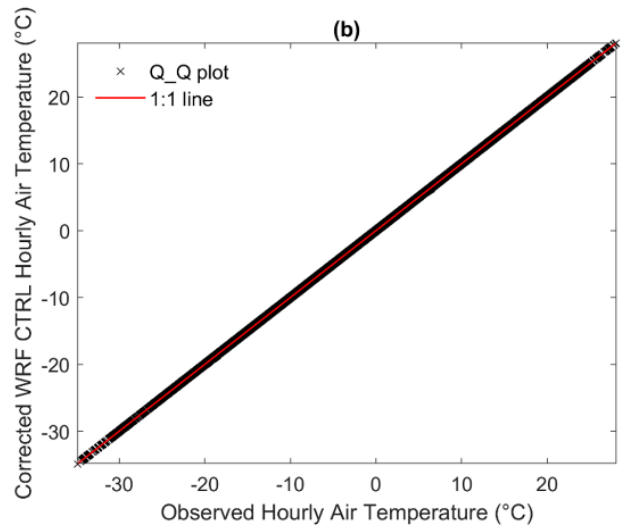

(d)

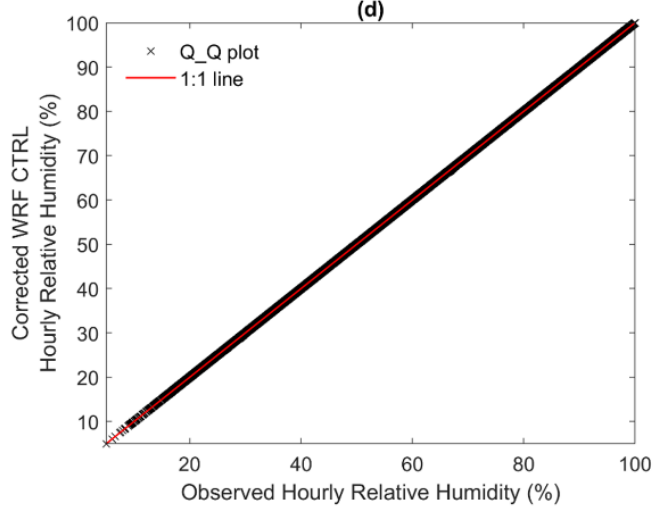

(f)

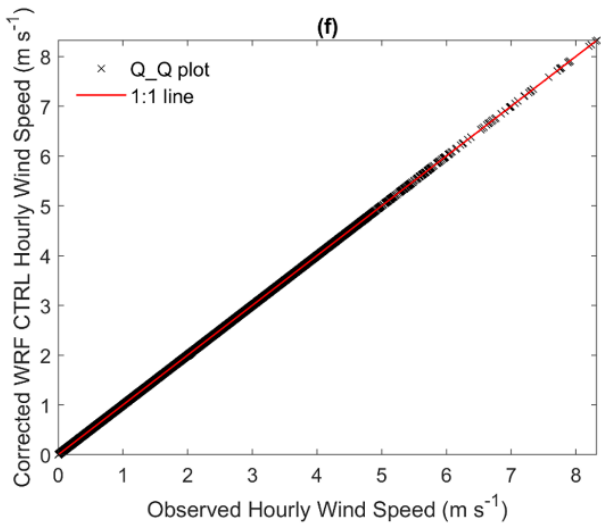

Figure S2. Quantile-quantile plots of observations and WRF CTRL outputs for Vista View station in MCRB: (a) WRF CTRL and observed air temperature, (b) corrected WRF CTRL and observed air temperature, (c) WRF CTRL and observed relative humidity (d) corrected WRF CTRL and observed relative humidity, (e) WRF CTRL and observed wind speed (f) corrected

5 WRF CTRL and observed wind speed, (g) WRF CTRL and observed incoming solar radiation (h) corrected WRF CTRL and observed incoming solar radiation, (i) WRF CTRL and observed precipitation (j) corrected WRF CTRL and observed precipitation. 
(a)

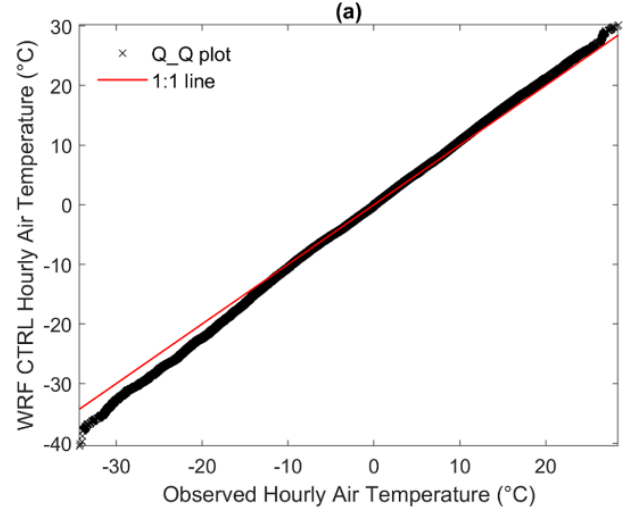

(c)

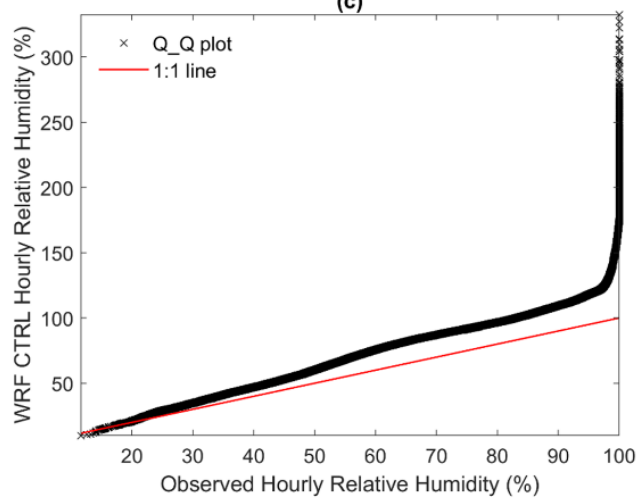

(e)

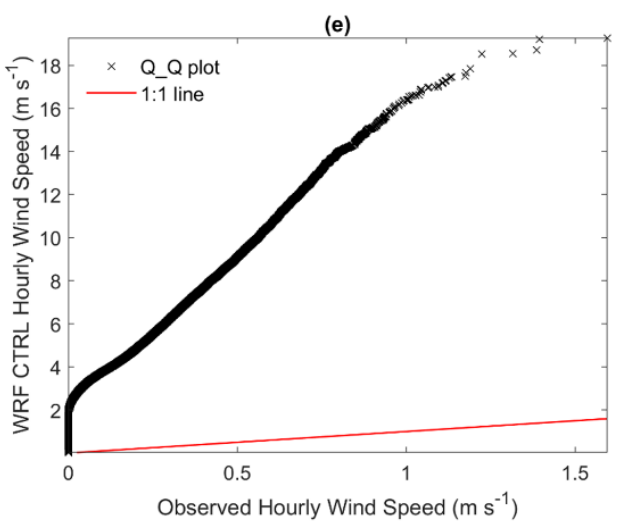

(b)

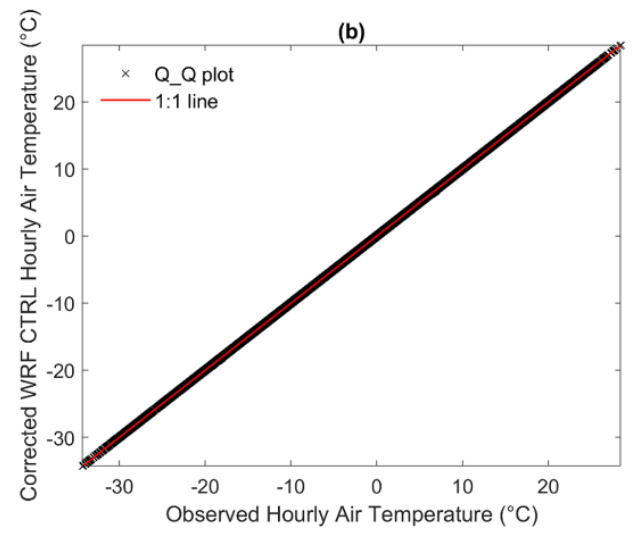

(d)
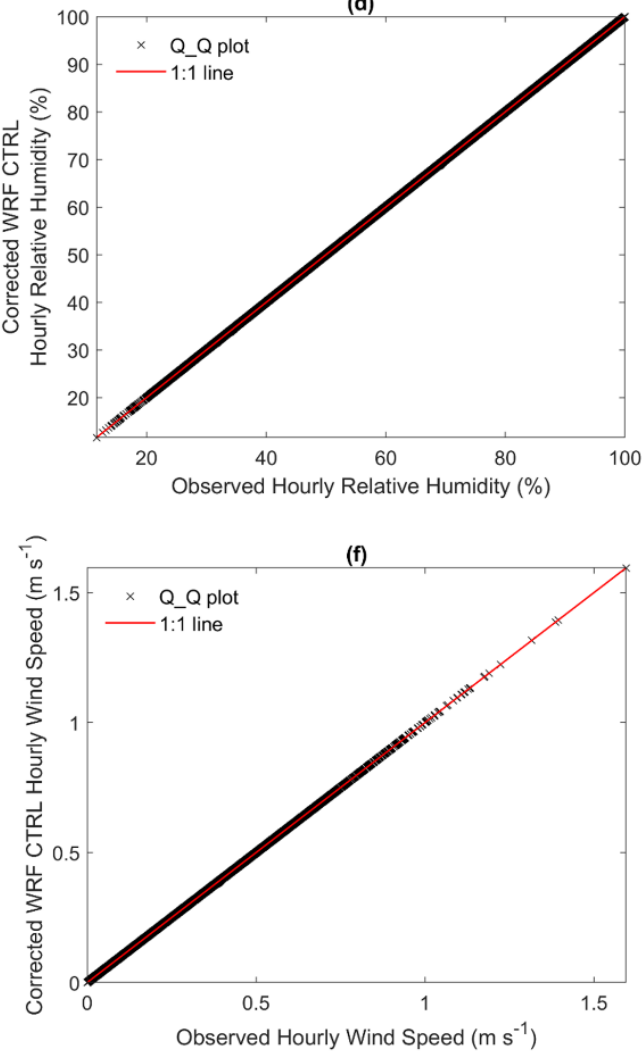

Figure S3. Quantile-quantile plots of observations and WRF CTRL outputs for Upper Forest station in MCRB: (a) WRF CTRL and observed air temperature, (b) corrected WRF CTRL and observed air temperature, (c) WRF CTRL and observed relative humidity (d) corrected WRF CTRL and observed relative humidity, (e) WRF CTRL and observed wind speed (f)

5 corrected WRF CTRL and observed wind speed, (g) WRF CTRL and observed incoming solar radiation (h) corrected WRF CTRL and observed incoming solar radiation, (i) WRF CTRL and observed precipitation (j) corrected WRF CTRL and observed precipitation. 
(a)

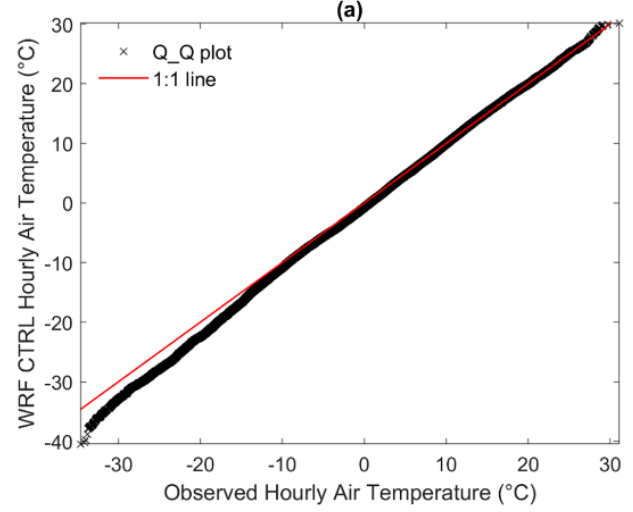

(c)

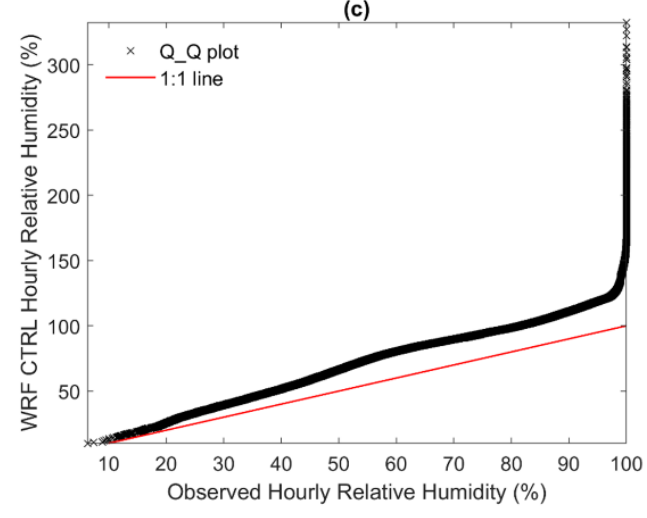

(e)

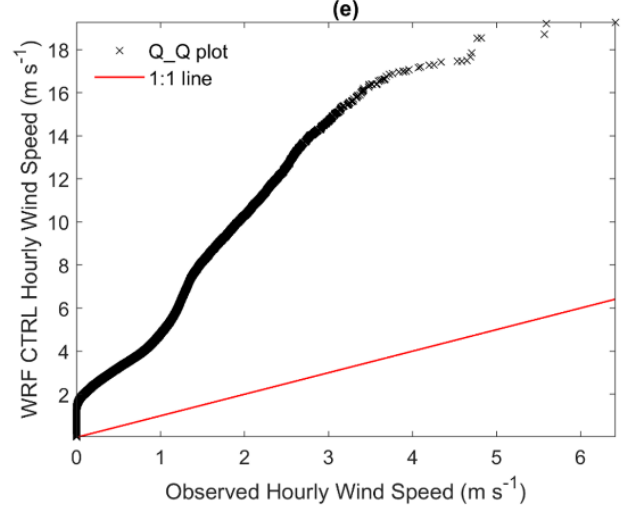

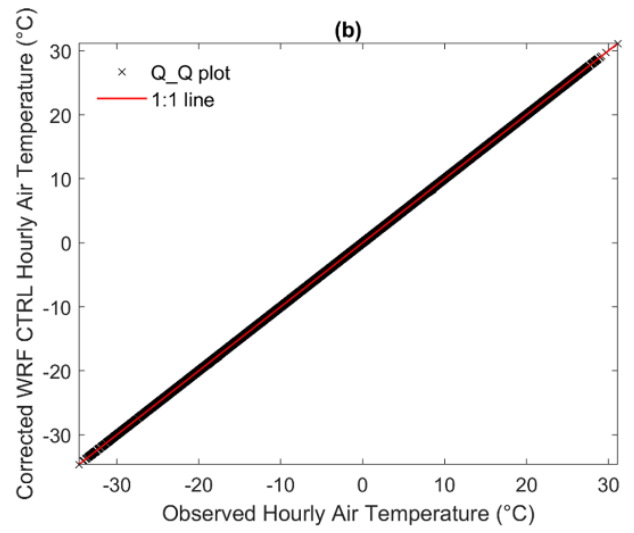

(d)
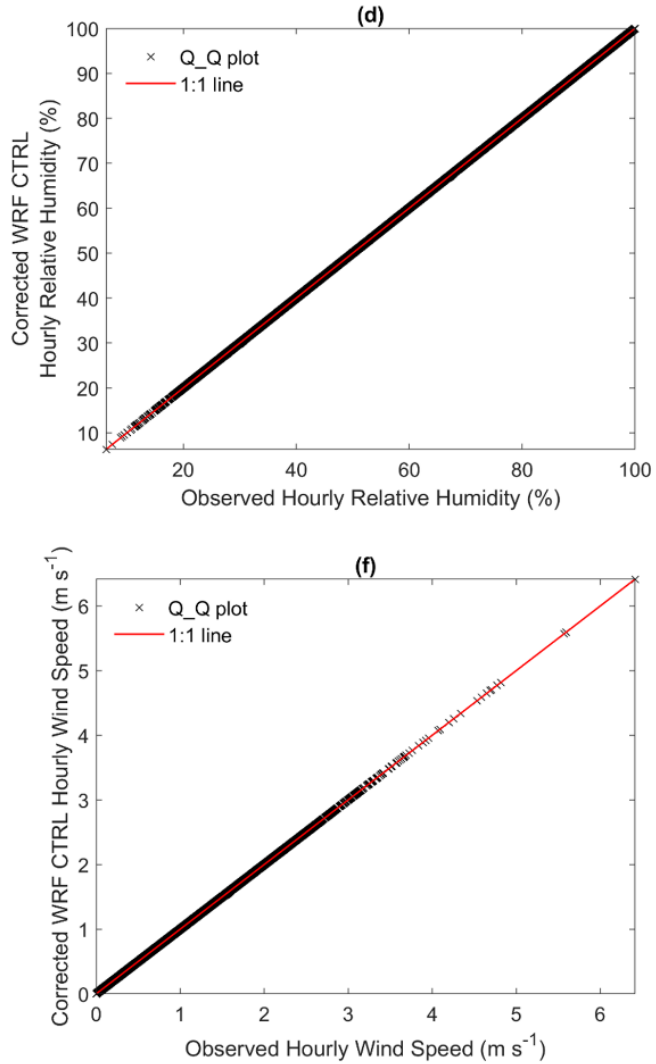

Figure S4. Quantile-quantile plots of observations and WRF CTRL outputs for Upper Clearing station in MCRB: (a) WRF CTRL and observed air temperature, (b) corrected WRF CTRL and observed air temperature, (c) WRF CTRL and observed relative humidity (d) corrected WRF CTRL and observed relative humidity, (e) WRF CTRL and observed wind speed (f)

5 corrected WRF CTRL and observed wind speed, (g) WRF CTRL and observed incoming solar radiation (h) corrected WRF CTRL and observed incoming solar radiation, (i) WRF CTRL and observed precipitation (j) corrected WRF CTRL and observed precipitation. 
(g)

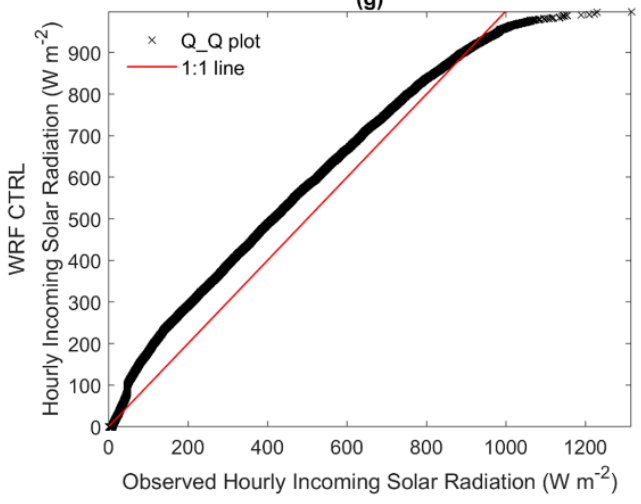

(i)

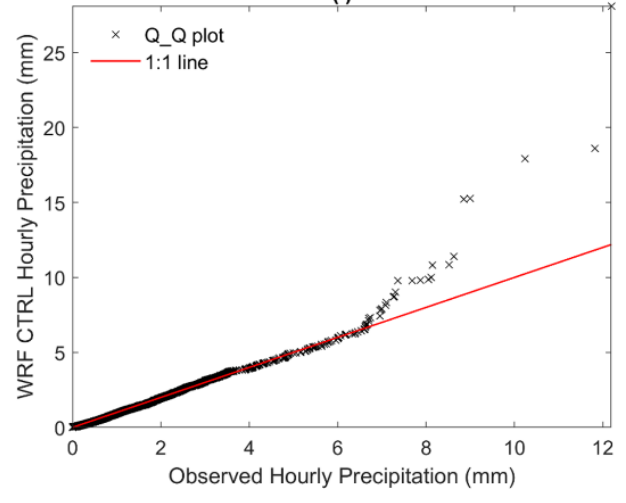

Figure S4. Continued. (h)
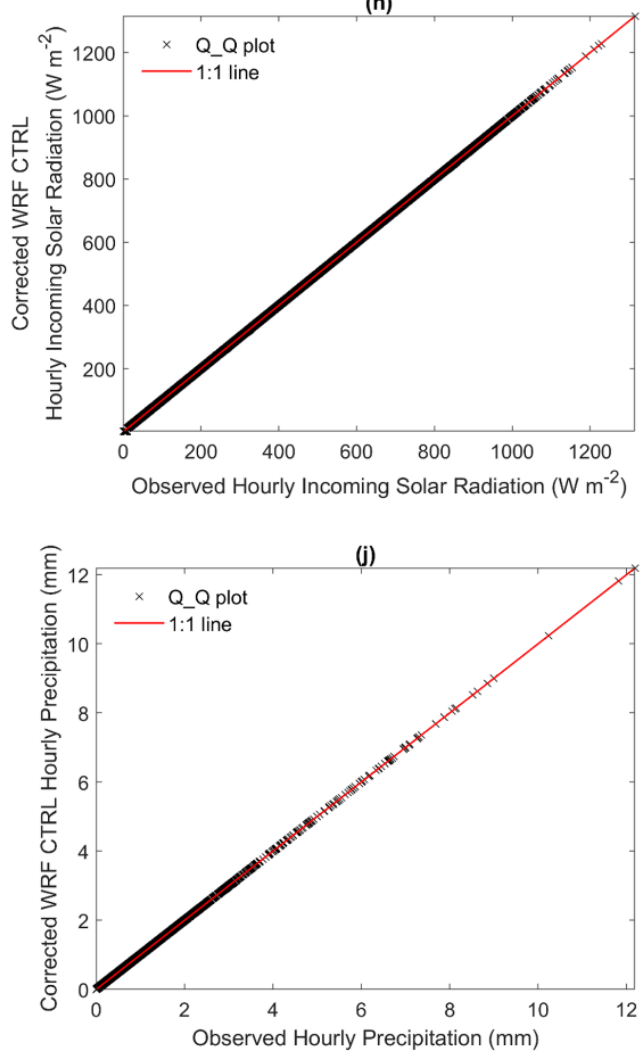